\title{
Creating One Time Password Programming for the Security of Data for Remote Location Treatment in Body Area Network
}

\author{
Prof. Pradeep Kumar \\ Associate Professor, Arya College of Engineering and ITArya College of Engineering and IT \\ Jaipur, Rajasthan, India \\ pmsuccess81@gmail.com
}

\begin{abstract}
Wireless body area network can manage patient through doctors through app on smart phones and security to data can be given by one-time password (OTP generation) like banking system. $6 \mathrm{G}$ protocols can make one time password more effective as all financial technology are dependent on one time password. Because transfer is mutual and one time password is best secure handshake between servers and containers are best security tempo for client server programmers. It depends on size of transfer means in the form of money in the transaction that size of container security code used for three way handshakes like third party or on the emergency for group client server model. Control logic is conditional enforcement of profitable rules to follow that is word access in computer science and that in the form of Intel processors till now but these Intel processors are in production in as general for as possible means one kind of Intel processor for whole world as processor generation paradigm. Can finance technological companies is eligible for creating more secure hardware for their trillions of transactions, I think word trillion is taken from word truth. Smartphone can handle data from sensor on body sensor through wireless with in distance limit and as data get uploaded it can reach to doctor by protecting it through OTP. In this as banking system can depend on OTP in the same way wireless health system may depend on this system.
\end{abstract}

Keywords: One-time password, Key Management, Body Area Network, Security, image as a key, C\#.

\section{Body area Network applications:}

Sensors in body area networks can take care about healthcare, control and automation, home and office, environmental monitoring, logistics and transportation, security and surveillance, tourism and leisure, education and training and entertainment. The BAN applications are broadly divided into following categories. Medical applications include many information of a patient and forward it to a monitoring centre for further analysis. BAN can also be used to help disable people as retina prosthesis chips can be implanted in the human eye to see at an adequate level. These days BANs are used for entertainment purpose, which includes 3D video and Games. Also, the BANs are used for sports, in which sensors in BAN can collect coordinates movements of different parts of the body and subsequently make the movement of a character in the game, e.g., moving soccer player or capturing the intensity of a ball in table tennis. At the end but not the least miscellaneous applications those include forgotten things monitoring, data file transfer and social networking applications.

\section{OTP Generation:}

In C\# I basically, hashed a time identifier (i.e. the current time in seconds divided by 30 - to get a long which is valid for the current 30-second interval). Then hashed this using my secret key as the SALT int onset $=$ hashBytes[19] \& $0 x f$;

int binaryCode $=$ (hashBytes[onset] \& $0 \times 7 f)<24$ 


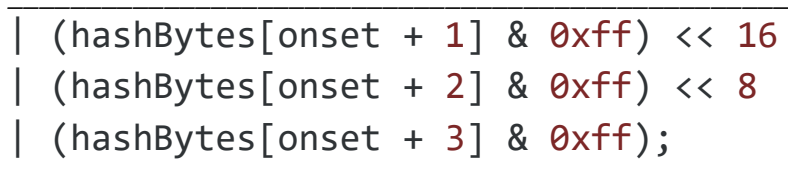

int otimep = binaryCode $\%$

(int)Math.Pow(10, 6);

return otp.ToString().PadLeft(6, ' 0 ');

\section{PROPOSED METHODOLOGY}

Security can be managed by one-time password in wireless body area network

STEP 1: Sensor can be attached to smartphone app plugged on body to upload the patient data.

STEP 2. On the same tome patient should apply to doctor at remote place after one-time password security check.

STEP 3. After data being uploaded, patient can get diagnosis after one-time password

STEP 4. Diagnosis and medicines name can be uploaded to online medical stores to dispatch medicines through courier to patient home.

STEP5. END

\section{CONCLUSION}

If bank can manage wealth through one-time password and we can manage health through onetime passwords in wireless body area network, in this we can fully utilised power of mobile technology. Even Location of the patient can be utilized for emergency reasons

\section{Reference:}

[1] Pradeep Kumar," Right Patient Data and Optimization Process Through Cryptographic Image as Key Using Genetic Algorithm in Body Area Network", ISSN (Online): 2347 - 4718, International Journal for Technological Research in Engineering Volume 5, Issue 9, May- 2018 